\title{
Commentary
}

\section{"Code of Practice and Guidance on Human Genetic Testing Services Supplied Direct to the Public". Advisory Committee on Genetic Testing}

\author{
John C K Barber
}

In the words of their Chairman, the Revd Dr John Polkinghorne, the Advisory Committee on Genetic Testing (ACGT) "is a nonstatutory advisory committee reporting to UK Health Ministers on developments in genetic testing". In this document, ${ }^{1}$ backed by the Department of Health, the Committee have produced the first guidelines for direct genetic testing of the public in this country. The main elements of the guidelines are that such testing should:

(1) Only be carried out in laboratories accredited to British Standard $\mathbf{5 7 5 0}$ (ISO9002) or Clinical Pathology Accreditation (UK) Ltd requirements. Continued BS5750 registration depends on satisfactory six monthly audits and CPA accreditation requires an external audit of 44 aspects of professional laboratory practice, annual re-registration, and a four yearly comprehensive reaccreditation assessment.

(2) Guarantee complete confidentiality of patient records apart from reports issued to GPs with patient consent.

(3) Ensure that test samples are stored for a minimum of three months and patient data for 12 months in case a result is queried. This is considerably less than the standards which apply in NHS laboratories and leaves an unrealistically short period of time over which results might in practice be challenged.

(4) Be limited to establishing carrier status for inherited recessive disorders and be specifically approved by the Committee before being made available.

(5) Not be provided to children under the age of 16 .

(6) $\mathrm{Be}$ accompanied by appropriate information in advance.

(7) Be provided only where opportunities for pre- and post-test genetic consultation are available without additional charge.

(8) Be notified to the patient's GP but only with the patient's written consent.

The Committee consulted widely in advance, placed the draft guidelines on the Internet and invited comments from all interested parties. Few would argue with the commend-

(7 Med Genet 1998;35:443-445)

Keywords: human genetic testing services; Advisory Committee on Genetic Testing ably brief guidelines contained in this document but this Code does, nevertheless, for the first time officially recognise and sanction genetic testing outside the context of a medical consultation.

At present, the vast majority of genetic testing in the UK takes place in 30 or so regional laboratories that are part of the National Health Service (NHS). They exist for the diagnosis, management and prevention of genetic disease, have close links with Clinical Genetic Services and frequently collaborate in research and development work likely to be of benefit to patients and their referring clinicians. The success of this network is recognised by the Committee and is widely regarded as a model of how genetic services can be effectively and responsibly provided. These centres are, however, constrained by the limited repertoire of tests funded by Health Authorities and it is only these tests which are available without charge to UK residents.

A potential conflict exists, therefore, between the medical profession which believes, not unreasonably, that testing should be focused on those whose phenotype or family history suggest a strong possibility of a genetic diagnosis, and the right of the individual person to buy the goods and services of their choice. Out of this has come the genetic testing by private laboratories for cystic fibrosis carrier status, as well as prenatal serum screening for Down syndrome in mothers who are less than 35 years old or who come from the many areas of the country where there is scepticism about the costs and benefits of serum screening. These tests are paid for by the people themselves, but can impact on the NHS in terms of the need for genetic advice or further prenatal chromosome tests. It is interesting to note, then, that the Code of Practice requires that members of the public are informed that genetic counselling is available, but that there is no requirement that a fee in respect of this component is added to the cost of the test.

A second group of patients frequently excluded from NHS provision are those seeking treatment for infertility. Some tests for the underlying cause of the infertility, such as Klinefelter syndrome or other chromosome abnormalities, may be covered while others,
District Hospital,

SP2 8BJ, UK

J C K Barber 
including mutations in the CF gene leading to congenital absence of the vas deferens or microdeletions of the DAZ (deleted in azoospermia) region, may not. Health Authorities are also unwilling to screen egg or sperm donors whose CF status is frequently determined by private companies or by NHS laboratories as private patients. Although these tests are taking place in the context of a medical consultation, it would be appropriate that the same standards of confidentiality and access to pre- and post-test genetic consultation apply to these patients as the ACGT now require of those offering tests directly to the public.

While the code restricts itself tightly to "carrier status for inherited recessive disorders in which an abnormal result carries no significant direct health implications for the customer", the reaction of the Press suggests that a much wider range of direct tests will need consideration in future. "Screen yourself by mail order" suggested The Times, ${ }^{2}$ while Medical Laboratory World went a stage further, announcing that "Cystic fibrosis tests for potential parents have been commercially available for some time, but now further tests, including those showing a predisposition for cancer, are on the cards for consumer use". ${ }^{3}$ A possible response to suggestions of this kind was provided by one ACGT member who was quoted in the same issue of The Times as saying that: "If somebody told us they were planning to offer tests for the breast cancer susceptibility gene BRCA1, we would have to think very hard". How might that thinking be influenced, however, if it had become clear that the choice for people with a family history of breast cancer was between no test at all through the NHS or paying for a test themselves?

Such demands can only be increased by the present growing inequalities in service provision within and between regions, and the widening gap between what is and what could be tested for. Compare, for instance, the person receiving a test for cancer predisposition without charge with the plight of a relative in a neighbouring region which might not fund the same test. Contrast the couple of dozen specific molecular genetic tests offered by the regional centres with the thousands of genetic conditions in which mutation analysis will shortly be possible. Now consider Health Authorities trying to balance limited funds against increasing demand at the same time as responding to national priorities. What prospect for the diagnosis, management, and prevention of all these other genetic conditions? What chance of this supposedly advanced society achieving the goal of providing to families with genetic conditions a quality of life as close as possible to that enjoyed by those without such conditions?

Meeting these challenges is a major task for the years' ahead, which laboratories are already facing up to. Firstly, they will be keen to adopt new technologies which promise to cut the cost of mutation detection by an order of magnitude over the next five years. These include the GeneChip (Affymetrix), ${ }^{4}$ multi- plex allele specific diagnostic assay (MASDA, Genzyme Genetics), ${ }^{5}$ and microplate assay diagonal gel electrophoresis (MADGE, Wessex Human Genetics Institute). ${ }^{6}$ Thus, while high start up costs may promote the geographical or functional fusion of laboratories of different sizes, new technologies should make it possible for the existing laboratory network to expand the range of tests offered within a given level of funding, provided that royalty costs are not exorbitant. Secondly, many laboratories have adjusted the balance between scientific and technical staff so that both quality standards and numbers of tests can be simultaneously raised without imposing additional changes. Thirdly, the declared intention of the recent UK government white paper is to recognise the existence of specialised services such as Genetics, and to ensure that all parts of the population have equal access to them. ${ }^{7}$ The promise of reducing inequality should not, however, be at the cost of a partial or mediocre service, but rather an opportunity to raise the level of provision for all. A clear mechanism for funding the transfer of research findings into new diagnostic tests will also be needed. For instance, we could already be identifying patients with factor $\mathrm{V}$ Leiden mutations who have an increased risk of thrombosis and patients with haemochromatosis mutations in whom the development of midlife liver cirrhosis and congestive heart failure is preventable. Analogous long term prevention programmes promise to deliver real improvements in health provided that the initial investment can be made before savings in other parts of the Health Service are recouped. ${ }^{8}$

Testing of this kind could be made available to medical practitioners or members of the public by both public services or private companies. NHS laboratories are part of an existing integrated structure, with a dedicated workforce who believe in collaborating to provide the best service for patients within the non-profit making ethos of the NHS. If they are to compete with private companies, however, they should also be allowed the freedom to reinvest surpluses in developing their own services as well as direct access to independent sources of funds for capital equipment.

In conclusion, it is essential that the public are not exploited and have confidence that genetics is being responsibly applied by laboratories working to high standards. Direct testing by private companies is already taking place, and the existence of the ACGT Code to regulate such testing is a welcome development. There is little doubt that private or NHS providers will be anxious to comply and that flagrant disregard of the Code would lead rapidly to legislation. There is also no doubt that the ACGT will have an expanding role in ensuring that the public can have confidence in the licensed genetic testing available in this country.

Copies of the Code of Practice, Guidance, and Proforma can be obtained from: Mrs M Straughan, The Secretariat, Advisory Committee on Genetic Testing, Department of 
Health, Room 401, Wellington House, 133155 Waterloo Road, London SE1 8UG, UK. Tel: 0171972 4017. Fax: 01719724196.

1 Advisory Committee on Genetic Testing. Code of practice and guidance on human genetic testing services supplied direct to the guidance on human genetic testing services supplied direct to the
public. London: Department of Health, September 1997.

2 Farrell S. Screen yourself by mail order. The Times, Wednesday 24 September 1997:6.

3 Genetic testing by post. Medical Laboratory World October 1997:3.

4 Castellino AM. When the chips are down. Genome Res 1997;7:943-6.
5 Shuber AP, Michalowsky LA, Nass GS, et al. High throughput parallel analysis of hundreds of patient samples for more than 100 mutations in multiple disease genes. Hum Mol Genet 1997;6:337-47.

6 Day INM, Humphries SE. Electrophoresis for genotyping: devices for high throughput using horizontal acrylamide gels (H-PAGE) and microtitre array diagonal gel electrophoresis (MADGE). Nature 1994;369:36-7.

7 The new NHS - modern, dependable" London: HMSO, Cmnd 3807, 1997.

8 Khoury MJ. Relationship between medical genetics and public health: changing the paradigm of disease prevention and the definition of a genetic disease. Am $\mathcal{f}$ Med Genet 1997;71:289-91. 